\title{
3 The relation of performance assessment to quality management
}

\author{
Janko Kersnik, Zalika Klemenc-Ketis
}

The definition of performance in the present EURACT Performance Agenda is the level of actual performance of physicians in clinical care and communication with patients in daily practice. Quality management is the evaluation of services provided and the results achieved as compared to accepted standards. The results of health care are compared with standard results and the deficiencies or problems defined serve to plan and introduce improvement actions. In quality management, this is called a PDCA cycle which has four steps:

- Plan what we are going to do. In this step we assess where we are, where we need to be, why this is important, and plan how to close the gap. Identify some potential solutions.

- Do try out or test the solutions (sometimes at a pilot level).

- Check to see if the countermeasures you tried out had the effect you hoped for, and make sure that there are no negative consequences associated with them. Assess if you have accomplished your objective.

- Act on what you have learned. If you have accomplished your objective, put controls into place so that the issue never comes back again. If you have not accomplished your objective, go through the cycle again, starting with the planning step.

\subsection{Relation of performance assessment to quality assessment}

The aim of any assessment in quality management is to see the level of quality of care (i. e. structure, processes and outcomes of care) in real practice settings. This serves as an indicator of quality level (used e.g. for ranking, certification, accreditation) and/or as a starting point to initiate necessary changes, which should lead to quality improvement, and as a benchmark to be compared with the assessment after change.

Performance assessment is therefore a core procedure of any quality assessment. However, any assessment in quality management has some inherent tendency to change behaviour, practice, and structure in order to get better outcomes. On the other hand, assessment in the educational realm is strictly for 
educational purposes in case of formative assessment, or for certification purposes in the case of summative assessment.

In quality management, there is no other way to assess quality of care than by some direct or surrogate way of observation of structure, process and outcomes. In education, knowledge, skills and attitudes as well as competence (see chapter 1) can be assessed by a variety of methods, which yield more or less reliable results with a good supposition that certified doctors will show good performance. Actual performance can be assessed during or at the end of the educational process and is best assessed in physicians' everyday practice settings. However, this is not considered as quality assessment as it is used solely for educational (CPD) or certification purposes. But when performance is assessed with the aim of improving the quality of physicians' work as a part of quality improvement (i. e. repeatedly in different stages of the PDCA cycle), it becomes a method for quality assessment and/or improvement (see also ${ }^{48}$ ). 